\title{
High-Speed Pulse Sequences through Semiconductor Optical Nonlinear Amplification and Buried Fiber Propagation
}

\author{
Ernesto M. M. Barrientos ${ }^{1,2}$ (D), Evandro Conforti ${ }^{2}$ (D) \\ ${ }^{1}$ Instituto Federal SP, Campinas, SP, Brazil, emartinb4@yahoo.com \\ ${ }^{2}$ University of Campinas, Unicamp, Campinas, SP,Brazil, conforti@ieee.org
}

\begin{abstract}
Microwave pulse bursts were obtained through optical domain intensity modulated microwave carriers, sliced by cascaded modulator technique, followed by non-linear semiconductor optical amplifier (SOA) pre-chirp. After 3 to $18 \mathrm{~km}$ of buried fiber propagation and photo detection, the microwave signals presented increased extinction ratio, up to $36 \mathrm{GHz}$, with pulse modulation windows from 166 to 1000 picoseconds, achieving rise times near to $10 \mathrm{ps}$.
\end{abstract}

Index Terms - electrooptic modulation, microwave photonics, pre-chirped pulses, semiconductor optical amplifier.

\section{INTRODUCTION}

The light amplification using semiconductors diodes was proposed by Basov et al. [1], using the electromagnetic energy generated by the recombination of electrical carriers in a p-n junction. For infrared wavelengths, gallium arsenide (Ga-As) was used for amplification [2]. However, useful Semiconductor Optical Amplifiers (SOAs) were achieved with multiple quantum-well (MQW) active regions and one-pass traveling wave SOAs were obtained using anti-reflections facets with small mirror reflectivity around $10^{-4}$ [3], [4]. Theoretical analysis of MQW-SOAs have been performed including gain compression in polarization insensitive devices [5], including injected current dynamics effects [6], [7]. In particular, when very short pulses are amplified, non-linear SOA effects of self-phase modulation (SPM) cause pulse spectral broadening and distortion [8]-[10]. Also, SPM introduces a pulse chirp (time-dependent instantaneous optical carrier frequency deviation) related to the SOA carrier lifetime, SOA saturation and gain [11]-[14]. The SOA chirping has been recently analyzed [15] including experimentally amplified $19 \mathrm{~ns}$ width individual pulses. This work introduces SOA amplification of fast microwave pulse bursts aiming applications of photonic techniques for arbitrary waveform generation (AWG) of microwave and millimetric signals [15]- [19]. They are obtained through optical domain amplitude modulated microwave carriers, sliced by a pulse-gated modulator technique [16]. The high power of the fast pulse bursts produces SOA gain saturation and chirping. So, proper SOA adjusted parameters produced nonlinear amplification of those short pulse's sequences (up to $10 \mathrm{ps}$ rise times), imbedded in an adjustable window (from $333 \mathrm{ps}$ to $1 \mathrm{~ns}$ ). The SOA amplified chirped pulses were transmitted in optical fibers (from 3 up to $18 \mathrm{~km}$ ) followed by photodetection. The tuned SOA and fiber parameters, followed by interaction of the pre-chirped pulses and fiber dispersion 
Journal of Microwaves, Optoelectronics and Electromagnetic Applications, Vol. 19, No. 3, September 2020 DOI: http://dx.doi.org/10.1590/2179-10742020v19i3880

enhanced the received signal format, extinction ratio, or rise-time. By adjusting the fiber length, the optical input pulse power and width, plus the SOA bias current, an improvement on the output pulse shape may be achieved as shown in the following sections. Section II presents a succinct theoretical background of the optical fast pulse SOA amplification, highlighting the optical chirp increment behavior (during the pulse rise and fall) and its interaction with the fiber dispersion. Section III shows the experimental setup description including the employed devices parameters. Section IV presents the experimental results with a discussion of how the output pulse shape improvement can be obtained. The conclusions are on Section V.

\section{THEORETICAL BACKGROUND}

The SOA amplification of fast pulses reaching the gain saturation produces nonlinear SPM effect. The time changing signal being amplified causes a dynamic carrier depletion, changing the SOA gain and the active region dielectric constant along the propagation axis $(z)$. Those complex effects were simplified by Agrawal and Olsson. For the case of unchirped Gaussian input pulse [8]:

$$
\operatorname{chirp}=\Delta v(\tau)=-(\partial \phi / \partial \tau) .(1 / 2 \pi)
$$

where $\Delta v$ is the pulse chirp, $\phi$ is the optical phase, and $\tau$ is the time moving with a reference frame. After same simplifying assumptions, the chirp increment, $\delta v$, after SOA pulse amplification is given by [8]:

$$
\delta v(\tau)=\Delta v_{\text {out }}(\tau)-\Delta v_{\text {in }}(\tau)=(\alpha / 4 \pi)(\partial h / \partial \tau)
$$

where $\Delta v_{\text {out }}$ is the SOA output signal chirp, $\Delta v_{\text {in }}$ is the SOA input signal chirp, $\alpha$ is the carrier induced index-change, $h$ is the SOA gain.

The derivative of the chirp increment, $C d$ is given by:

$$
C d=\partial[\delta v(\tau) / \partial \tau]=(\alpha / 4 \pi)\left(\partial^{2} h / \partial \tau^{2}\right)
$$

At the pulse beginning (zero amplitude), the SOA gain is at the its maximum value, $h=G_{0}$, where $G_{0}$ is the small signal SOA gain. Assuming instantaneous response of the SOA gain $h$, as soon as the pulse rises, the SOA gain decreases due to saturation. The chirp increment of (2) attains a negative (red-shift) value, since the gain time derivative is negative $(\partial h / \partial \tau<0)$. As the pulse continues to rise, the gain decreases even more (due to the increased saturation effect). When the maximum pulse intensity is reached, the SOA gain attains its minimum value (the gain second derivative $\partial^{2} h / \partial \tau^{2}>0$ ), the first derivative is zero ( $\partial h / \partial \tau=0$ ), and no chirp is produced. During the pulse fall, the SOA gain begins to increase $(\partial h / \partial \tau>0)$ since the pulse signal is dropping, and the SOA saturation is decreasing. So, the chirp reaches a positive value.

However, in practice the SOA behavior is intricate. The SOA gain does not have an instantaneous response. The solution of (2) for a pulse rise time much faster than the SOA carrier lifetime, predicts a sharper pulse trailing edge, and a slower decay in the remaining pulse [8]. Also, the output pulse spectrum has a red shift value whose peak decreases several GHz. At the pulse beginning (SOA bias of 
$70 \mathrm{~mA}$ ), an experimental chirp value of $\delta v=-30 \mathrm{GHz}$ was obtained for a $19 \mathrm{ps}$ amplified pulse width [15]. Later, the chirp increment reached a value around $\delta v=-12 \mathrm{GHz}$ at the maximum pulse amplitude peak. And at the minimum amplitude (where the pulse ends) the chirp was around $\delta v=+6 \mathrm{GHz}$. The SOA overall red shift was $-12 \mathrm{GHz}$ [15]. Also, the chirp nonlinearity is more intense when the pulse intensity and the SOA bias increase [15]. Likewise, the chirp increment $\delta v$ and its time variation $C g$ depend on the SOA manufacture parameters, the SOA saturation level and bias current, the relation of the pulse width and the SOA carrier lifetime, plus the pulse shape and pre-chirp.

However, since the chirp increment $\delta v$ (frequency variation) changes during the pulse rise and fall, the pulse shape can be improved after fiber propagation, since the fiber dispersion also changes with the light instantaneous carrier frequency. A standard $10 \mathrm{~km}$ fiber at $1550 \mathrm{~nm}$ has a dispersion of 170 $\mathrm{ps} / \mathrm{nm}$, or $2.1 \mathrm{ps} / \mathrm{GHz}$ of the instantaneous carrier frequency. A typical output SOA pulse has a negative chirp $\delta v$ at pulse rising and a positive chirp at pulse decaying. During the hole pulse duration, the total chirp variation can be $36 \mathrm{GHz}$ [15] or more, and this frequency change is converted in a signal time variation (compression or enlargement) due to the fiber dispersion. Therefore, a $20 \mathrm{GHz}$ chirp (frequency variation) during the pulse rising might reach up to $42 \mathrm{ps}$ of pulse format variation in a 10 $\mathrm{km}$ fiber. By adjusting the fiber length, the optical input pulse power and width, plus the SOA bias current, an improvement on the output pulse shape may be achieved as shown in the following sections. The numerical analysis is out of the scope of this work.

\section{EXPERIMENTAL SETUP}

The generation of the embedded optical pulse train employed a cascaded single-drive Mach-Zehnder modulators (MZM) [16]. As shown in Fig. 1, the $40 \mathrm{GHz}$ synthesized microwave generator (MG) drives (at $17 \mathrm{dBm}$ ) the first optical intensity modulator (MZM1), with a $3 \mathrm{~dB}$ bandwidth equal to $40 \mathrm{GHz}$. The MZM1 has zero chirp and insertion loss of $5 \mathrm{~dB}$. The technique permits an arbitrary signal instead of the sinusoidal signal. However, optical intensity modulated harmonic signals are easier to analyze and were chosen here.

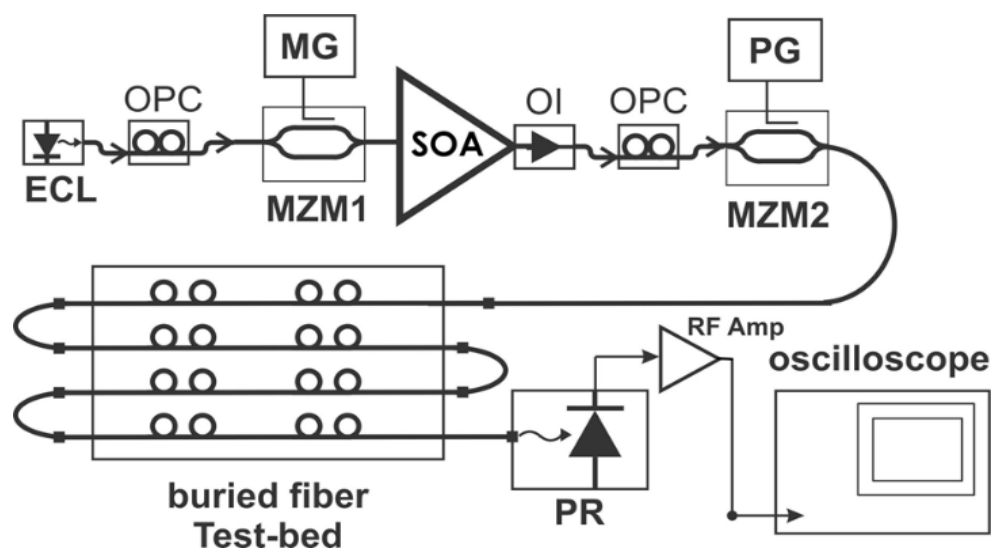

Fig. 1. Block diagram of the setup with external cavity laser (ECL), optical polarization controller (OPC), microwave generator (MG), Mach-Zehnder modulator (MZM), non-linear semiconductor optical amplifier (SOA) to produce chirped signal, optical isolator (OI), pulse generator (PG), photo receiver (PR), and oscilloscope. Optical paths have thicker lines.

Brazilian Microwave and Optoelectronics Society-SBMO received 11 Feb 2020; for review 13 Feb 2020; accepted 17 June 2020 
Journal of Microwaves, Optoelectronics and Electromagnetic Applications, Vol. 19, No. 3, September 2020 DOI: http://dx.doi.org/10.1590/2179-10742020v19i3880

The light carrier was provided by a continuous wave (CW) $1550 \mathrm{~nm}$ external cavity laser (ECL), 100 $\mathrm{kHz}$ linewidth, followed by an optical polarization controller (OPC). The modulated optical output carrier ( $3 \mathrm{dBm})$ was amplified by a SOA model XN-OEC-1550, ultra-nonlinear, from CIP (UK), with cavity length of $2 \mathrm{~mm}$, optical output saturation power (Psat) of $15 \mathrm{dBm}$, and maximum gain around $38 \mathrm{~dB}$ (small signals, @500 mA). The device was biased at $220 \mathrm{~mA}$ to provide static optical gain around $12 \mathrm{~dB}$, assuring a SOA operation under saturation regime to provide a proper SPM effect. No optical bandpass filter is inserted after the SOA since the chirped pulse could have its bandwidth altered. The SOA was followed by an optical isolator (OI), an OPC, and a cascaded second modulator (MZM2), zero chirp, with $3 \mathrm{~dB}$ bandwidth equal to $40 \mathrm{GHz}$. A 30 ps rise time pulse generator (PG) drives MZM2 to a level of $2.5 \mathrm{~V}$ (close to $\mathrm{V} \pi$ ) converting the MZM2 as a gated switch (on-off operation). With a proper synchronization of MG and PG, the gated modulator embedded the MG chirped sinusoidal signal in a variable length square window. A 50\% duty cycle was chosen with a window length of 333 ps up to $1 \mathrm{~ns}$.

The embedded chirped signal was propagated in a test-bed of buried fibers using the "Kyatera Network". This optical buried fiber facility connects optical communication laboratories throughout the State of São Paulo, Brazil. The results here employed a $1.5 \mathrm{~km}$ cable of 16 standard fibers with chromatic dispersion of $17 \mathrm{ps} /(\mathrm{nm} . \mathrm{km})$ at $1550 \mathrm{~nm}$, with FC-APC connectors at each end. Finally, an 8 ps rise-time photonic receiver (PR) followed by a $40 \mathrm{GHz}$ sampling scope was used, for the measurements before and after fiber propagation. The optical signal was also tested with an optical spectrum analyzer with sub $\mathrm{nm}$ resolution.

\section{EXPERIMENTAL RESULTS}

A typical result obtained with the setup of Fig. 1 is shown in Fig. 2, where a $12 \mathrm{GHz}$ microwave carrier was embedded in a gate-width of 250 ps with a pulse repetition frequency (PRF) of $2 \mathrm{~Gb} / \mathrm{s}$. Given a period of $83.3 \mathrm{ps}$ for the $12 \mathrm{GHz}$ sine wave, only $3 \mathrm{RF}$ cycles can fit the 250 ps gate, as noted in Fig. 2. Also, the dotted line signal is the chirped pulse just after the SOA. The continuous line of Fig. 2 shows the same signal after the fiber propagation of $3 \mathrm{~km}$. Note the typical distortion due to SPM [8] of the dotted line chirped signal. After $3 \mathrm{~km}$ of fiber propagation, a discrete improvement on the signal format can be observed.

The same signals of Fig. 2 are shown in Fig. 3, but the fiber length is now $9 \mathrm{~km}$. A better signal format now appears (continuous line) with an improvement on the extinction ratio and on the signal format. In addition, a good suppression of the microwave oscillation was obtained outside the 250 ps gate, but small microwave energy is still presented. The uncomplete suppression is due to insufficient extinction of MZM2. Also, the three pulses did not reach the zero level and the insufficient extinction ratio might be related to both MZM1 and MZM2 limited bandwidth. 


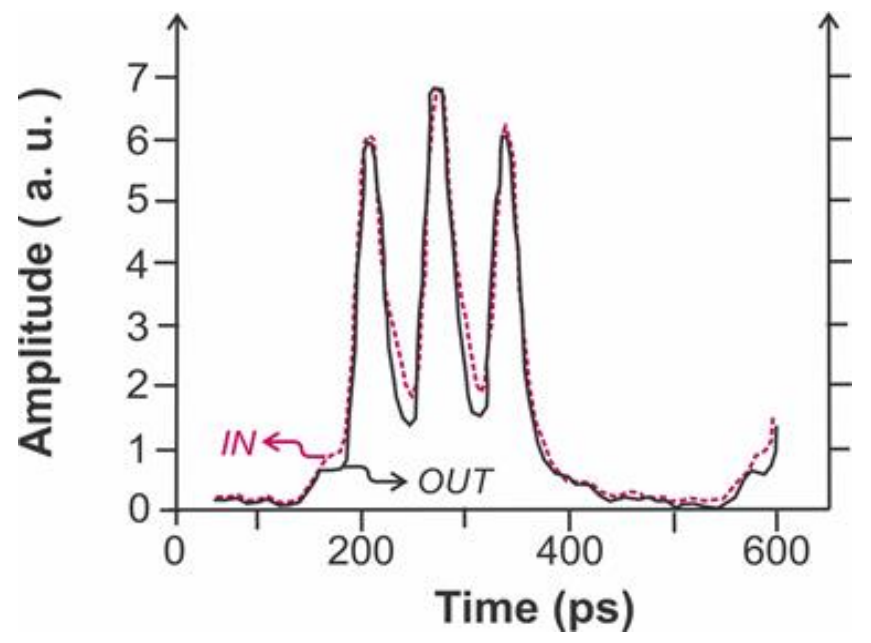

Fig. 2. Photo-detected signals for a $12 \mathrm{GHz}$ microwave carrier after the SOA (dotted line) and after $3 \mathrm{~km}$ of fiber propagation (continuous line).

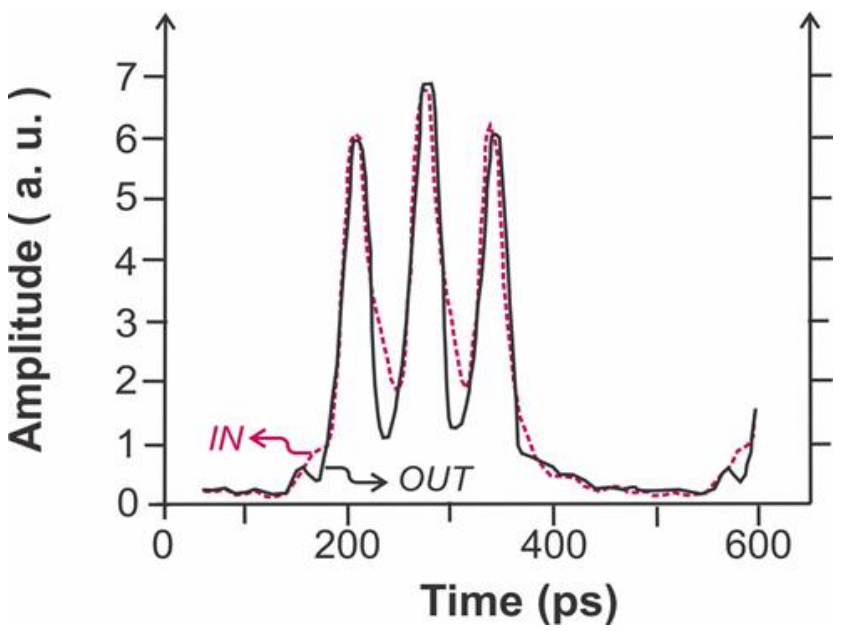

Fig. 3. Photo-detected signals for a $12 \mathrm{GHz}$ microwave carrier after the SOA (dotted line) and after $9 \mathrm{~km}$ of fiber propagation (continuous line).

The results for the same signals of Fig. 2 are shown in Fig. 4 for an overall fiber length of $18 \mathrm{~km}$. Note the higher extinction ratio of the propagated signal (continuous line). However, the received signal has distortions during the rising time of the internal pulses, shown by the letters A and B in Fig. 4. This behavior might be due to an over correction of the pulse format, since the longer fiber has greater dispersion and additional attenuation given by the 18 connectors at the fiber endings.

Similarly, several results (not shown) present a similar behavior, denoting the existence of an interaction of the pulse (with embedded chirp due to the SOA non-linear behavior) and the fiber length, since the dispersion at $1550 \mathrm{~nm}$ in a standard fiber is around $17 \mathrm{ps} / \mathrm{nm} . \mathrm{km}$. Indeed, the SOA chirp can reach over $100 \mathrm{GHz}$ for short pulses [8]. However, the SOA chirp behavior is a complex feature and the analysis is out of the scope here. In general, the chirp intensity and its time format depend on the SOA itself, the bias current, the optical input pulse intensity, width, and format. 


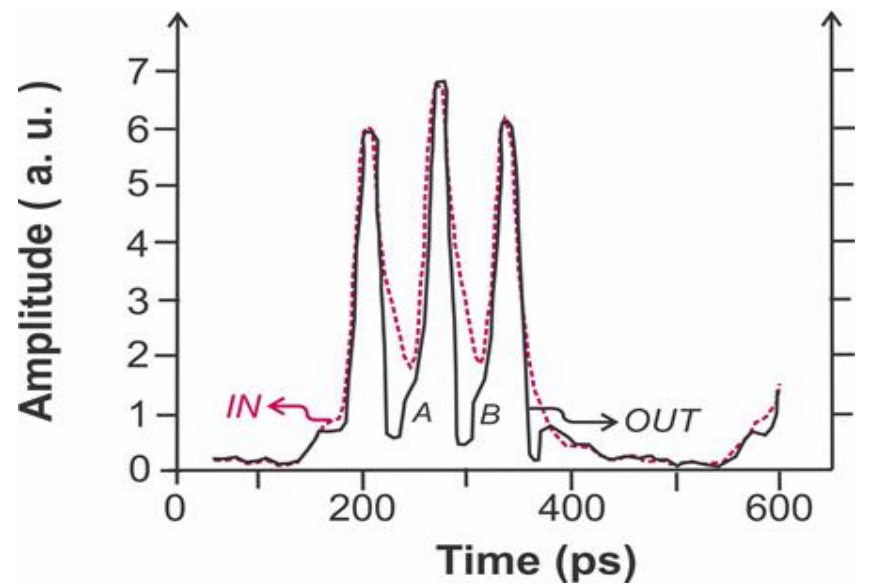

Fig. 4. Photo-detected signals for a $12 \mathrm{GHz}$ microwave carrier after the SOA (dotted line) and after $18 \mathrm{~km}$ of fiber propagation (continuous line).

An experimental result for a $20 \mathrm{GHz}$ microwave carrier and a $250 \mathrm{ps}$ embedding window is shown in Fig. 5. The dotted line is the SOA chirped signal and the continuous line after $9 \mathrm{~km}$ fiber propagation. In this case, the improvement of the extinction ratio can be noted. However, the microwaves outside the embedding window were not suppressed entirely. This behavior might be related to the MZM2 gated switch insufficient action to suppress the higher $20 \mathrm{GHz}$ modulated carrier signal inside it.

The results for a $12 \mathrm{GHz}$ microwave signal inside an embedded window of 166 ps are shown in Fig.6 for $9 \mathrm{~km}$ of fiber propagation. The dotted line presents pulse distortions, shown by letters $\mathrm{C}$ and $\mathrm{D}$. Also, the letters A and B represents the $10 \%$ and $90 \%$ signal levels of the continuous line after propagation. Those levels A and B were used to calculate the signal rise time as shown in Fig. 6. Also note the half cycle calculation in Fig. 6.

Several measurements have been made with the microwave signals going from $12 \mathrm{GHz}$ up to $36 \mathrm{GHz}$, including the embedded windows from $166 \mathrm{ps}$ up to $500 \mathrm{ps}$, for fiber lengths of $3.0 \mathrm{~km}, 9.0 \mathrm{~km}$, and 18 $\mathrm{km}$.

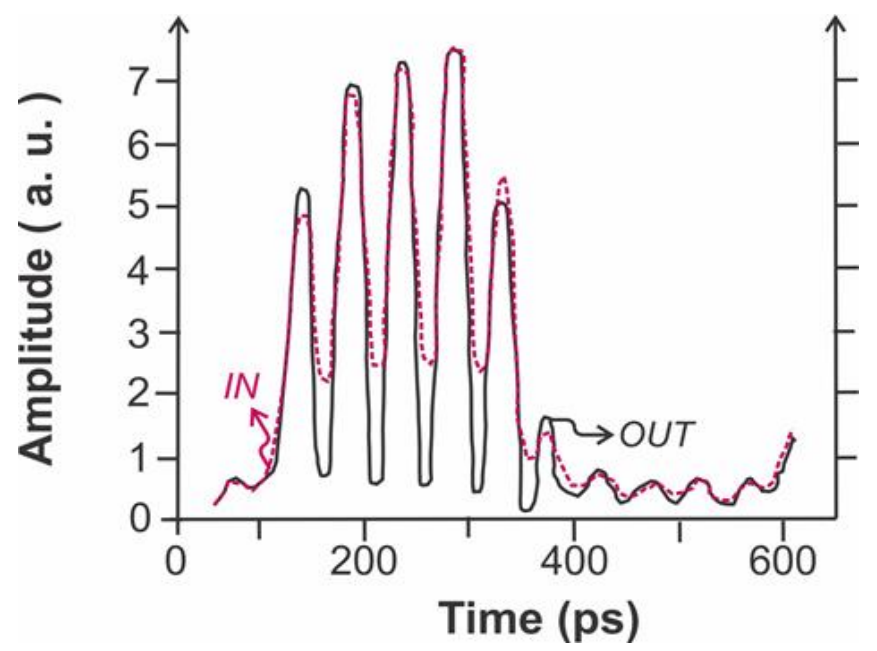

Fig. 5. Photo-detected signals for a $20 \mathrm{GHz}$ microwave carrier and $250 \mathrm{ps}$ window after the SOA (dotted line) and after 9 $\mathrm{km}$ of fiber propagation (continuous line). 


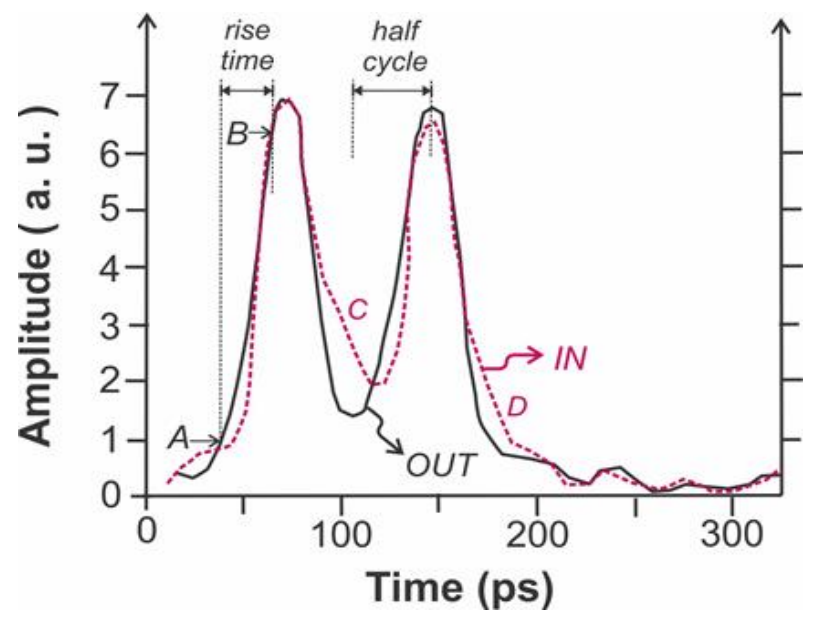

Fig. 6. Photo-detected signals for a $12 \mathrm{GHz}$ microwave carrier in a $166 \mathrm{ps}$ window after the SOA (dotted line) and after 9 $\mathrm{km}$ of fiber propagation (continuous line), with rise time and half cycle estimation.

The results of the mean rise and fall time of the same pulse are presented in Fig. 7 as a function of the half cycle. The theoretical values were calculated for an ideal sinusoidal wave (dotted line). The results for the chirped signal (no fiber propagation) is shown by a continuous line. The signal propagated mean rise-fall time after $3 \mathrm{~km}, 9 \mathrm{~km}$, and $18 \mathrm{~km}$ are shown by the dotted-point line, the dotted-x line, and the dotted-o line, respectively. The $9 \mathrm{~km}$ line results showed improvements in relation to the no fiber propagation. The $18 \mathrm{~km}$ result is worse than no propagation and might be attributed to an over correction of the pulse format. The microwave signal and optical spectra were also measured (results not shown here).

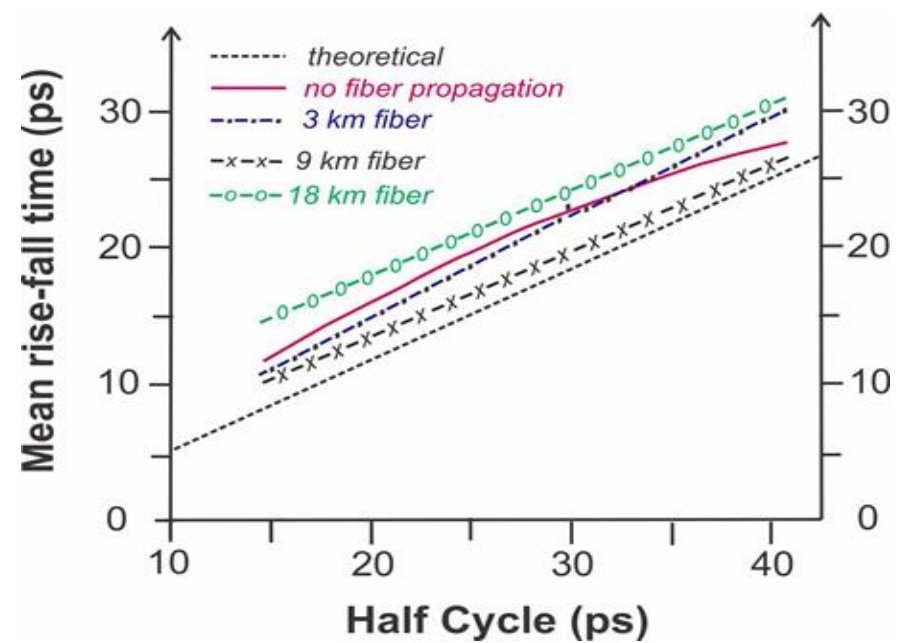

Fig. 7. Results of the mean rise and fall time of several tested pulses as a function of the half cycle, including theoretical results (dotted line), no fiber propagation (continuous line), and after fiber propagation; $3 \mathrm{~km}$ (dotted-point line); $9 \mathrm{~km}$ (dotted-x line); and 18 km (dotted-o line).

\section{CONCLUSION}

A technique for the generation of fast chirped optical pulsed waveforms, including the format and extinction ratio measurements after the fiber propagation has been described. The chirped pulses have been produced by amplification and proper parameter adjustments of a nonlinear SOA with $2 \mathrm{~mm}$ length cavity. The experimental setup successfully regenerated gate embedded window microwave signals, Brazilian Microwave and Optoelectronics Society-SBMO received 11 Feb 2020; for review 13 Feb 2020; accepted 17 June 2020 
with microwave carriers up to $36 \mathrm{GHz}$, minimum pulse windows of $166 \mathrm{ps}$, with reformatted pulse rise time approaching $10 \mathrm{ps}$. Further works are related to the extinction ratio improvement of the microwave signal, tests with different dispersions fibers and SOAs, and proper numerical evaluation.

\section{ACKNOWLEDGEMENT}

This work was supported in part by the Brazilian Agencies São Paulo Research Foundation (FAPESP - grants 2015/24517-8, 2015/50063-4, and 2007/56024-4), and National Council for Scientific and Technological Development (CNPq - grants 301409/2017-0 and 402923/2016-2).

\section{REFERENCES}

[1] N. G. Basov, O. N. Kroknin, and Y. M. Popov, "Production of Negative-Temperature States in p-n Junctions of Degenerate Semiconductors," Soviet Physics JETP, vol. 13, pp. 1320-1321, 1961.

[2] R. N. Hall, G. E. Fenner, J. D. Kingsley, T. J. Soltys, and R. O. Carlson, "Coherent Light Emission from GaAs Junctions," Physical Review Letters, vol. 9, pp. 366-368, 1962.

[3] G. Eisenstein, "Semiconductor Optical Amplifiers," IEEE Circuits and Devices Mag., vol. 5, pp. 25-30, 1989.

[4] K. Obermann, I. Koltchanov, K. Petermann, S. Diez, R. Ludvig, and H. G. Weber, "Noise Analysis of Frequency Converters Utilizing Semiconductor-Laser Amplifiers," IEEE J. Quantum Electron., vol. 33, pp. 81-88, 1997.

[5] A. Reale, A. D. Carlo, and P. Luigi, "Gain Dynamics in Traveling-Wave Semiconductor Optical Amplifiers," in IEEE J. Select. Topics Quantum Electron., vol. 7, no. 2, pp. 293-299, 2001.

[6] T. Sutili, E. M. M. Barrientos, A. C. Bordonalli, C. M. Gallep, and E. Conforti, "Computational Model and Parameter Extraction of High Speed Semiconductor Optical Amplifier Space Switches," Journal of Microwaves, Optoelectronics and Electromagnetic Applications, vol. 17, no. 4, pp. 646-660, 2018.

[7] P. Rocha, C. M. Gallep, T. Sutili, and E. Conforti, "Calibration of TLM Model for Semiconductor Optical Amplifier by Heuristic Parameters' Extraction," Journal of Microwaves, Optoelectronics and Electromagnetic Applications, vol. 17, no. 2, pp. 260-267, 2018.

[8] G.P. Agrawal, and N.A. Olsson, "Self-phase modulation and spectral broadening of optical pulses in semiconductor laser amplifiers", IEEE J. Quantum Electr., vol. 25, no. 11, pp. 2297-2306, 1989.

[9] G. P. Agrawal, "Effect of gain dispersion on ultrashort pulse amplification in semiconductor optical amplifier", IEEE $J$. Quantum Electr., vol. 27, no. 6, pp. 1843-1849, 1991.

[10] J. Chung, N. Kikuchi, and S. Sasaki, "Generation of transform-limited optical pulses using a combination of a gainswitched diode laser and a semiconductor optical amplifier," IEEE Photon. Technol. Lett., vol. 7, no. 8, pp. 860-862, 1995.

[11] I. W. Marshall, and D. M. Spirit, "Observation of large-pulse compression by a saturated traveling wave semiconductor laser amplifier," in. CLEO, Anaheim, Ca, USA, paper TUM64, 1988.

[12] L. E. Y. Herrera, R. M. Ribeiro, V. B. Jabulka, and J. P. von der Weid, "Optical generation and transmission of linearly chirped microwave pulses with time-bandwidth product," in. OFC, San Diego, Ca, USA, paper M1H.2, 2018.

[13] D. Hadass, V. Mikhelashvili, G. Eisenstein, A. Somers, S. Deubert, W. Kaiser, J. P. Reithmaier, A. Forchel, D. Finzi, and Y. Maimon, "Time-resolved chirp in an InAs-InP quantum-dash optical amplifier operating with 10 Gbps data," Applied Physics Letters, vol. 87, no. 2, pp. 021104, 2005.

[14] M. Matsuura, N. Iwatsu, K. Kitamura, and N. Kishi, "Time-Resolved Chirp Properties of SOAs Measured with an Optical Bandpass Filter," IEEE Photonics Technology Letters, vol. 20, no. 23, pp. 2001-2003, Dec. 2008.

[15] M. J. Connelly, J. Romero-Vivas, J., L. Krzczanowicz, A. Meehan, , "Dynamic power and chirp measurements of amplified 19 ps pulses in traveling-wave and reflective semiconductor optical amplifiers using a linear pulse characterization technique," Opt Quant Electron, vol. 51, no. pp. 248-1-248-10, 2019.

[16] E. M. M. Barrientos, C. S. Castelli, A. M. O. Ribeiro, and E. Conforti, "Microwave Carrier Modulated by Fast Synchronous Pulsed Transitions using Electro-Optical Gating Modulators," 2009 IEEE MTT-S International Microwave Symposium Digest, Boston, MA, USA, June 5 -11, paper TUPF-5, 2009. DOI 10.1109/MWSYM.2009.5165711

[17] W. Liu and J. Yao, "Photonic Generation of Microwave Waveforms Based on a Polarization Modulator in a Sagnac Loop," J. Lightwave Technol. vol. 32, pp. 3637-3644, 2014.

[18] W. Zhai, A. Wen, and D. Shan, "Photonic Generation and Transmission of Frequency-Doubled Triangular and Square Waveforms Based on Two Mach-Zehnder Modulators and a Sagnac Loop”, J. Lightwave Technol. vol. 37, pp. 1937$1945,2019$.

[19] M. A. Romero and S. Arismar Cerqueira, "Microwave-Photonics and WDM-PON Fronthaul for 5G Mobile Systems: Research Activities in Brazil," 2019 49th European Microwave Conference (EuMC), Paris, France, pp. 268-271, 2019. 